\title{
FOCUS EDITORIAL
}

\section{Critical illness: the brain is always in the line of fire}

\author{
Martin Smith ${ }^{1,2^{*}}$ and Geert Meyfroidt ${ }^{3,4}$
}

๑๐ 2017 Springer-Verlag Berlin Heidelberg and ESICM

Life-threatening systemic insults or diseases often affect the brain. In critically ill patients, acute brain dysfunction manifests in several ways including reduced consciousness, coma, or delirium. The pathophysiology is complex, incompletely understood, and may relate to critical illness-related inflammatory changes, neurotransmitter imbalances, or failure of adequate energy substrate delivery [1], as well as to the applied treatments, pharmacological neurotoxicity, or the hostile ICU environment (Fig. 1) [2].

Delirium warrants some emphasis because it is the most common manifestation of critical illness-related brain dysfunction [3]. In a recent study of 420 critically ill mechanically ventilated adult patients, $54 \%$ experienced delirium for at least 1 day (median delirium duration 2 days, interquartile range 1-4 days) [4]. The hippocampus and frontal cortex, both areas that are extremely vulnerable to metabolic and hypoxic-ischemic insults, appear to be predominantly involved. Triggers of ICU delirium include predisposing factors (older age, previous cognitive impairment, arterial hypertension), precipitating factors (mainly related to the severity of critical illness), and factors that can potentially be treated or avoided including medications (benzodiazepines, opioids, and anticholinergics), pain, sepsis, fever, metabolic disturbances (dysglycemia, dysnatremia), and environmental factors (sleep deprivation, noisy environment).

An acute encephalopathy is reported in up to $70 \%$ of patients with sepsis. Systemic insults, such as hemodynamic changes leading to impaired cerebral perfusion, severe hypoxemia, endocrine dysfunction, or fever, are aggravated by blood-brain barrier dysfunction,

\footnotetext{
*Correspondence: martin.smith@ucl.ac.uk

${ }^{1}$ Neurocritical Care Unit, The National Hospital for Neurology and Neurosurgery, University College London Hospitals, Queen Square, London, UK

Full author information is available at the end of the article
}

impairment of the cerebral microcirculation, microglial activation by pro- and anti-inflammatory cytokines and chemokines, and altered neurotransmission [5]. In some cases this can lead to cerebral edema, ischemia, or seizures.

The electroencephalography (EEG) findings of sepsisrelated encephalopathy are characterized by a diffuse cortical dysfunction with generalized EEG slowing and the presence of theta and delta waves [3]. Seizures in sepsis are often non-convulsive in nature and require EEG monitoring for their identification. A recent single-center study identified a high incidence of non-convulsive seizures (11\%) and periodic discharges (25\%) in a cohort of patients with sepsis-related alterations in mental status, although this was not associated with a higher mortality or disability rates at 6 and 12 months after discharge [6]. However, loss of EEG reactivity to external stimulation, a sign of severe encephalopathy, was associated with increased mortality in this patient cohort. Large, prospective studies are required to determine the outcome effects of EEG abnormalities in the critically ill septic patient and whether their treatment modulates outcomes.

Critical illness-related brain dysfunction is associated with worse short-term outcomes, such as longer duration of mechanical ventilation and increased ICU and hospital lengths of stay, but its consequences can also extend beyond the ICU episode. Previously assumed to be completely reversible, it is now clear that sepsis-associated encephalopathy can lead to permanent neurocognitive impairments and might also increase the brain's susceptibility to neurodegenerative disease including dementia [5]. In the BRAIN-ICU (bringing to light the risk factors and incidence of neuropsychological dysfunction in ICU survivors) study, $40 \%$ of 821 critically ill adult patients with respiratory failure or shock had global cognitive dysfunction at 3 and 12 months after hospital discharge

\section{Springer}




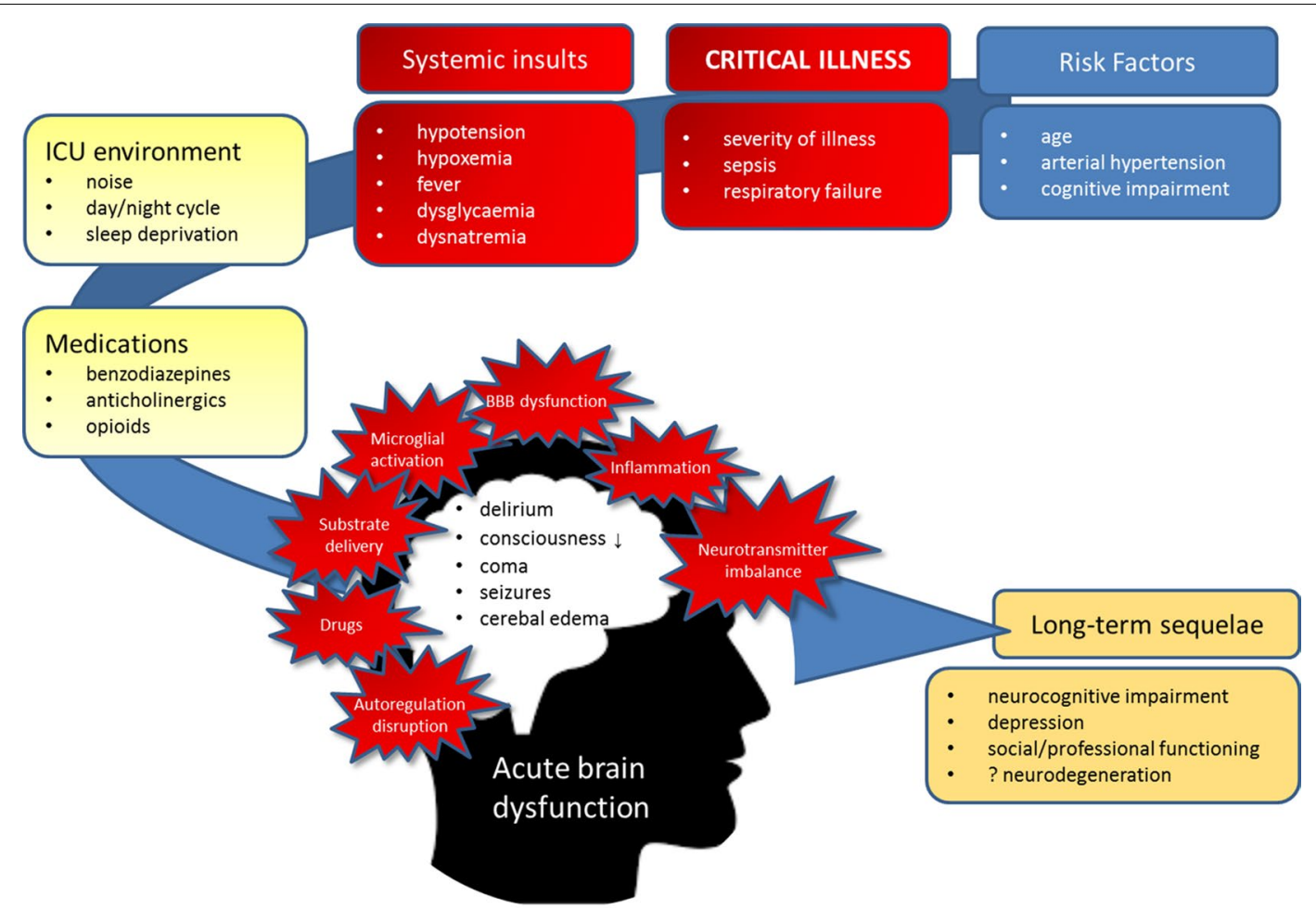

Fig. 1 Risk factors and contributors to critical illness-acquired brain dysfunction (blue, red, and yellow boxes), mechanisms of brain injury (red explosions), and long-term neurological sequelae (orange boxes). The arrow indicates the interactions between the multiple interconnected insults and mechanisms that contribute to the development of critical illness-related brain dysfunction, and which are associated with adverse outcome effects including long-term cognitive dysfunction

[7]. In $26 \%$ of patients, 3 -month cognitive function scores were similar to those reported in mild Alzheimer's disease, and this poor level of function persisted in $24 \%$ of those in whom cognitive assessments were available at 1 year. Although the prevalence of post-ICU cognitive impairment declines over time, it can be long-lasting in some patients. In one study, $20 \%$ of survivors of ARDS had residual cognitive impairment 5 years after hospital discharge [8]. However, the exact scale of the problem is uncertain because of the large variability in studies investigating cognitive outcomes after critical illness. A recent systematic review reported a wide range (4-62\%) of postICU cognitive impairment in adult patients followed up between 2 and 156 months [9]. This variation is likely related to the different definitions of cognitive impairment in individual studies, different durations of followup, and failure to adjust for pre-ICU cognitive function and other comorbidities.

Post-ICU cognitive impairment occurs in multiple domains but is most pronounced in areas of memory, executive functioning, and attention, suggesting that critical illness results in diffuse injury to the brain [10]. This is supported by the EEG findings of diffuse encephalopathy and neuroimaging correlates [3]. Critical illness is frequently associated with structural and functional neuroimaging abnormalities that can persist for months and years, and which may correlate with poor cognitive outcomes [11]. Structural brain abnormalities include global and focal atrophy (particularly in the hippocampus and superior frontal lobes in patients with delirium), white matter lesions suggesting sepsis-related increased blood-brain barrier permeability, and cortical and subcortical lesions likely related to cerebral hypoperfusion [3]. Reduced white matter connectivity in the corpus callosum and internal capsule has also been demonstrated in ICU survivors. There are currently only limited data investigating neuroimaging abnormalities in ICU survivors, and further studies are required to establish the role of neuroimaging in predicting long-term outcomes and guiding rehabilitation in ICU survivors [11].

Modulation of microglial activation, prevention of alterations in blood-brain barrier permeability, and use of antioxidants represent potential future therapeutic strategies, but current management focuses on prevention and treatment of causative factors. Minimization of harm from therapies for supporting critically ill patients is possibly more relevant to the brain than to any other organ system [1]. Much attention has focussed on 
medications, particularly benzodiazepines, as important modifiable risks factors. In a cohort study in 1112 critically ill adults, benzodiazepine administration was associated with an increased risk of transition from an awake, clear-conscious state to delirium on the following day, and continuous infusion of the drug was associated with a higher risk of transition compared to intermittent bolus [12]. Such findings strengthen the argument for reliance on non-benzodiazepine sedative agents in critically ill patients.

Dysglycemia, including hyperglycemia, hypoglycemia, or marked variability in glucose concentration, is ubiquitous in the acutely ill and may contribute to the development of critical illness-related brain dysfunction [13]. Prolonged hyperglycemia in the context of critical illness is a particular risk factor. The exact mechanisms are speculative but, like in sepsis, are multifactorial and likely related to increased blood-brain barrier permeability, oxidative stress, and microglia activation. While glycemic control with insulin infusion during critical illness has variable effects on overall outcomes, it might hold promise to preserve brain cell viability and minimize the risk of brain dysfunction. A meta-analysis of glycemic control in neurocritical care patients suggested improved neurological outcomes in those treated with insulin therapy despite an increased risk of hypoglycemia [14]. While hypoglycemia should always be promptly corrected in the critically ill, overcorrection must be avoided because excessive glucose reperfusion may worsen neurologic injury [13].

Early identification of patients with an increased risk of developing delirium might facilitate more effective preventative strategies. In a large international, prospective cohort study a multiple logistic regression analysis was used to develop and validate an early prediction (E-PRE-DELIRIC) model for delirium [15]. Using nine easily assessable variables, E-PRE-DELIRIC is able to stratify patients at ICU admission for the risk of delirium throughout their whole ICU episode with high discrimination. Further studies are required to refine and further validate risk prediction models which can ultimately be used to guide clinical decision-making as well as to riskstratify participants in therapeutic clinical trials of delirium prevention and management [16]. Notwithstanding the importance of predicting patients at high risk of ICU-acquired delirium, regular monitoring for delirium onset using standard screening tools cannot be overemphasized. Multicenter randomized clinical trials are also needed to determine which interventions are most effective in reducing the incidence and duration of critical illness-induced brain dysfunction, and the impact of this on short- and long-term outcomes.

Whatever the cause of admission to the ICU, there is increasing awareness of the importance of the adverse effects of critical illness and its treatment on both shortand long-term brain function. Care protocols that aim to optimize cerebral physiology are routinely applied during the management of acute brain injury [17], and similar approaches are likely to be relevant more widely. Critical illness always puts the brain in the line of fire and it has been suggested that a brain-oriented approach should be a unifying concept in the management of all critically ill patients [1].

\footnotetext{
Author details

${ }^{1}$ Neurocritical Care Unit, The National Hospital for Neurology and Neurosurgery, University College London Hospitals, Queen Square, London, UK. ${ }^{2}$ UCLH/UCL National Institute for Health Research Biomedical Research Centre, London, UK. ${ }^{3}$ Department of Intensive Care Medicine, University Hospitals Leuven, Leuven, Belgium. ${ }^{4}$ Department of Cellular and Molecular Medicine, KU Leuven-University of Leuven, Leuven, Belgium.
}

\section{Compliance with ethical standards}

\section{Conflicts of interest}

MS is a Section Editor of Intensive Care Medicine. MS is part funded by the University College London Hospitals University College London National Institute for Health Research Biomedical Research Centre. GM is funded by the Research Foundation, Flanders (FWO) as a senior clinical investigator. No other conflicts declared.

Received: 14 March 2017 Accepted: 31 March 2017

Published online: 6 April 2017

\section{References}

1. Tasker RC, Menon DK (2016) Critical care and the brain. JAMA 315:749. doi:10.1001/jama.2016.0701

2. Zaal IJ, Spruyt CF, Peelen LM et al (2013) Intensive care unit environment may affect the course of delirium. Intensive Care Med 39:481-488. doi:10.1007/s00134-012-2726-6

3. Hughes CG, Patel MB, Pandharipande PP (2012) Pathophysiology of acute brain dysfunction. Curr Opin Crit Care 18:518-526. doi:10.1097/ MCC.0b013e328357effa

4. Mehta S, Cook D, Devlin JW et al (2015) Prevalence, risk factors, and outcomes of delirium in mechanically ventilated adults. Crit Care Med 43:557-566. doi:10.1097/CCM.0000000000000727

5. Widmann CN, Heneka MT (2014) Long-term cerebral consequences of sepsis. Lancet Neurol 13:630-636. doi:10.1016/S1474-4422(14)70017-1

6. Gilmore EJ, Gaspard N, Choi HA et al (2015) Acute brain failure in severe sepsis: a prospective study in the medical intensive care unit utilizing continuous EEG monitoring. Intensive Care Med 41:686-694. doi:10.1007/ s00134-015-3709-1

7. Pandharipande PP, Girard TD, Jackson JC et al (2013) Long-term cognitive impairment after critical illness. N Engl J Med 369:1306-1316. doi:10.1056/NEJMoa1301372

8. Herridge MS, Moss M, Hough CL et al (2016) Recovery and outcomes after the acute respiratory distress syndrome (ARDS) in patients and their family caregivers. Intensive Care Med 42:725-738. doi:10.1007/ s00134-016-4321-8

9. Wolters AE, Slooter AJC, van der Kooi AW, van Dijk D (2013) Cognitive impairment after intensive care unit admission: a systematic review. Intensive Care Med 39:376-386. doi:10.1007/s00134-012-2784-9

10. Hopkins RO, Wade D, Jackson JC (2017) What's new in cognitive function in ICU survivors. Intensive Care Med 43:223-225. doi:10.1007/ s00134-016-4550-x 
11. Hopkins RO, Suchyta MR, Beene K, Jackson JC (2016) Critical illness acquired brain injury: neuroimaging and implications for rehabilitation. Rehabil Psychol 61:151-164. doi:10.1037/rep0000088

12. Zaal IJ, Devlin JW, Hazelbag M et al (2015) Benzodiazepine-associated delirium in critically ill adults. Intensive Care Med 41:2130-2137. doi:10.1007/s00134-015-4063-z

13. Sonneville R, Vanhorebeek I, den Hertog HM et al (2015) Critical illnessinduced dysglycemia and the brain. Intensive Care Med 41:192-202. doi:10.1007/s00134-014-3577-0

14. Ooi YC, Dagi TF, Maltenfort M et al (2012) Tight glycemic control reduces infection and improves neurological outcome in critically ill neurosurgical and neurological patients. Neurosurgery 71:692-702. doi:10.1227/ NEU.0b013e3182631eb4
15. Wassenaar A, van den Boogaard M, van Achterberg T et al (2015) Multinational development and validation of an early prediction model for delirium in ICU patients. Intensive Care Med 41:1048-1056. doi:10.1007/ s00134-015-3777-2

16. Salluh JIF, Latronico N (2015) Making advances in delirium research: coupling delirium outcomes research and data sharing. Intensive Care Med 41:1327-1329. doi:10.1007/s00134-015-3864-4

17. Wijdicks EFM, Menon DK, Smith M (2015) Ten things you need to know to practice neurological critical care. Intensive Care Med 41:318-321. doi:10.1007/s00134-014-3544-9 